\title{
Detailed proteome mapping of newly emerged honeybee worker hemolymph and comparison with the red-eye pupal stage
}

\author{
Tomas Erban ${ }^{1}$, Karel Harant ${ }^{2}$, Martin Kamler $^{3}$, Martin Markovic ${ }^{1}$, Dalibor TiterA ${ }^{3}$ \\ ${ }^{1}$ Laboratory of Proteomics, Crop Research Institute, Drnovska 507/73, Praha 6-Ruzyne CZ-16106, Czechia \\ ${ }^{2}$ Faculty of Science, Charles University in Prague, Prague, Czechia \\ ${ }^{3}$ Bee Research Institute at Dol, Libcice nad Vltavou, Czechia
}

Received 18 September 2015 - Revised 24 January 2016 - Accepted 15 February 2016

\begin{abstract}
The honeybee, Apis mellifera, undergoes complete metamorphosis before transitioning to the adult stage. The newly emerged individual and the red-eye pupa stage are well defined and easily recognizable in the time life cycle honeybee and, therefore, very useful for studying physiological and developmental factors. We analyzed in detail the hemolymph proteome of newly emerged honeybee worker using 2D-E-MS/MS (pI 3-10 and 4-7). The comparison of identical hemolymph volumes $(20 \mu \mathrm{L}$ per $2 \mathrm{D}$-E) for newly emerged bee and red-eye pupa revealed a dramatic decrease in the number of spots (qualitative changes) and overall protein quantity during the non-feeding stage. The results increase our knowledge about honeybee metamorphosis during the non-feeding period and clarify previous findings regarding particular proteins. The results will be useful for future comparative physiological, developmental, and host-pathogen studies on individual or population level.
\end{abstract}

\section{Apis mellifera / hemolymph / metamorphosis / hexamerin / ferritin}

\section{INTRODUCTION}

The honeybee, Apis mellifera L., is a holometabolous insect that undergoes complete metamorphosis before transitioning to the adult stage. The development time of European honeybee workers is typically 21 days. The pupal stage begins with the molt from the last larval stage, a few days after a brood cell was sealed. During this period, the body is reconstructed and, finally, the capping is eaten away and the adult emerges (Winston 1987). The just/newly emerged honeybee, like the redeye pupa stage (Erban et al. 2014), is a well-defined, easily recognizable developmental stage in the

Electronic supplementary material The online version of this article (doi:10.1007/s13592-016-0437-7) contains supplementary material, which is available to authorized users.

Corresponding author: T. Erban, arachnid@centrum.cz Manuscript Editor: Klaus Hartfelder honeybee life cycle and, therefore, very useful for studying physiological and developmental factors that can be caused by aging, entomopathogens (such as viruses), bacteria, Nosema, the hemolymph-sucking mite Varroa, or the influence of pesticides.

Similarly to human blood, insect hemolymph contains important indicators of the physiological state of the body. Since the protein composition of the honeybee hemolymph varies between developmental stages, hemolymph proteins can be used to investigate caste differences and developmental changes (Chan et al. 2006; Chan and Foster 2008). Changes in the hemolymph protein composition from larval to pupal development stages of $A$. mellifera ligustica were studied by Woltedji et al. (2013), and a proteomic approach has been used to study hemolymph proteome changes comparing A. mellifera and A. cerana during worker brood development, including pupae (Feng et al. 2014). We previously described the hemolymph 
of the red-eye pupal stage at the proteome level (Erban et al. 2014). In addition, honeybee hemolymph proteins of different developmental stages were detected in the ectoparasite Varroa destructor (Erban et al. 2015).

In this study, we analyzed in detail the hemolymph of newly emerged honeybee workers using two-dimensional gel electrophoresis (2D-E) combined with a matrix-assisted laser desorption/ ionization (MALDI) time-of-flight (TOF)/TOF proteomics approach. In addition to the proteomic 2D-E-MS/MS mapping of emerged honeybees, we aimed to compare these results with the hemolymph protein composition of the red-eye pupal stage described in our previous study (Erban et al. 2014). The results indicated that at the end of metamorphosis/time of emergence, the honeybee hemolymph became depleted of proteins and only a few proteins remained at a higher concentration. A dramatic change in the hemolymph protein composition was identified when comparing redeye pupae with newly emerged bees. In addition to these comparative results, we believe that the description of the methodical approach presented here, i.e., simple direct proteomic analysis without sample processing producing quality $2 \mathrm{D}$-Es or comparison of same hemolymph volumes between stages, will also be of interest for further studies.

\section{MATERIALS AND METHODS}

\subsection{Reagents}

Iodoacetamide (IAA; Cat No. 57670), dithiothreitol (DTT; Cat No. 43817), agarose (Cat No. A7431), ammonium bicarbonate (ABC; Cat No. A6141), trifluoroacetic acid (TFA; Cat No. 91707), acetonitrile (ACN; Cat No. 34998), 37.5:1 acrylamide/bisacrylamide solution (Cat No. 01709), N,N, $N^{\prime}, \mathrm{N}^{\prime}-$ tetramethylethylenediamine (TEMED; Cat No. T9281), Bradford reagent (Cat No. B6916), methanol LC-MS CHROMASOLV $^{\circledR}$ (Cat No. 34966), $\alpha$-cyano4-hydroxycinnamic acid (CHCA; Cat No. 70990), and HPLC/MS-grade water were obtained from SigmaAldrich (St. Louis, MO, USA). Acetic acid was obtained from Lach-ner (Neratovice, Czechia). Ultra-pure sodium dodecyl sulfate (SDS; Cat No. 2326.2), glycine (Cat No. 3908.2), and Tris base (TRIS; Cat No. 4855.3) were obtained from Carl Roth (Karlsruhe, Germany). DeStreak Rehydration solution (Cat No. 17-6003-19), IPG buffer pH 3-10 (Cat No. 17-6000-87), IPG buffer pH 4-7 (Cat No. 17-6000-86), ammonium persulfate (APS; Cat No. 17-1311-01), Immobiline ${ }^{\text {TM }}$ Dry Strip pH 3-10, $13 \mathrm{~cm}$ (Cat No. 17-6001-14), Immobiline Dry Strip pH 4-7, $13 \mathrm{~cm}$ (Cat No. 17-6001-13), and PhastGel $^{\mathrm{TM}}$ Blue R (Cat No. 17-0518-01) were obtained from GE Healthcare Life Sciences (GE) (Uppsala, Sweden). Trypsin (Cat No. v5111) was obtained from Promega (Fitchburg, WI, USA).

\subsection{Biological samples}

The newly emerged honeybees used in this study were collected from monitored Varroa -free A. mellifera carnica colony at the Bee Research Institute at Dol in Czechia. For hemolymph collection, individual honeybees were removed with forceps as they were chewing through the cell caps. Thus, the bees did not eat before collection, nor were they affected by factors associated with the environment outside the hive. On the other hand, the bees were not influenced by long absence of the queen or hive because we collected the samples within $20 \mathrm{~min}$ after the comb was taken from the hive. Hemolymph was immediately retrieved from the dorsal vessels using a glass capillary, after puncturing the abdominal intersegmental membrane between the 4 th and 5 th tergites. Approximately $20-40 \mu \mathrm{L}$ of transparent hemolymph was collected from each individual. The hemolymph was transferred from the glass capillaries to microcentrifuge tubes and immediately frozen on dry ice, or mixed in volumes of either 20 or $30 \mu \mathrm{L}$ with DeStreak Solution containing $0.5 \%$ IPG buffer $\mathrm{pH} 3-10$ or $4-7$ and then frozen on dry ice. The samples were stored at $-80{ }^{\circ} \mathrm{C}$ in a deep freezer (Thermo, Waltham, MA, USA) until use. Prior to preparation of this paper, we performed more than 50 2D-E separations (Coomassie or fluorescence stained and 13- or 7cm strips; data not shown) of emerged worker bee hemolymph from different hives. The 2D-E images presented in this study were collected in April and originated from a single colony. Six 2D-Es were selected for protein identifications in this study. 


\subsection{Two-dimensional gel electrophoresis}

A total of 20 or $30 \mu \mathrm{L}$ of hemolymph from a single honeybee was used for each 2D-E experiment. We did not process the hemolymph before analysis, analogous to Erban et al. (2014), as the samples mixed with DeStreak containing $0.5 \%$ IPG buffer (pH 3-10 or 47) were directly used for isoelectrofocusing (IEF). IEF was performed on an Ettan IPGphor 3 (GE). The separation was performed in $13-\mathrm{cm}$ ceramic strip holders and Immobiline dry strips ( $\mathrm{pH} 3-10$ or 4-7). The separation program was as follows: (1) step, $30 \mathrm{~V}, 10 \mathrm{~h}$ (active rehydration); (2) step, $500 \mathrm{~V}, 500 \mathrm{Vh}$; (3) grad, $1000 \mathrm{~V}, 800 \mathrm{Vh}$; (4) grad, $6000 \mathrm{~V}, 15,000 \mathrm{Vh}$; and (5) step, $6000 \mathrm{~V}, 16,000 \mathrm{Vh}$. The IEF ran for $19 \mathrm{~h}$ and a total of $32,600 \mathrm{Vh}$. Following IEF, the strips were equilibrated for $15 \mathrm{~min}$ in an equilibration buffer containing DTT, followed by $15 \mathrm{~min}$ in a buffer with IAA. The strips were placed over a $14 \%$ SDS-PAGE gel and fixed with $1 \%$ agarose. Electrophoresis was run at a constant voltage in a cooled apparatus (SE 600 Ruby, GE). The gels were fixed (40\% methanol, $10 \%$ ice acetic acid, and $50 \% \mathrm{ddH}_{2} \mathrm{O}$ ) overnight and stained using $0.02 \%$ PhastGel Blue R. Unused fixing solution was used for destaining. The results were visualized with the G:BOX (Syngene, Cambridge, UK).

\subsection{MALDITOF/TOF protein identification}

Spots (0.5- to $1-\mathrm{mm}$ inner diameter) were selected from the Coomassie-stained gels and subjected to 4800 Plus MALDI TOF/TOF (Applied Biosystems/MDS SCIEX; Foster City, CA, USA) analysis. The sample preparation procedures and subsequent protein identification of trypsin digests followed the methodology described by Erban et al. (2014). The spectra were searched against the non-redundant (nr) NCBI database using MASCOT 2.2 (Boston, MA, USA). The database search criteria were as follows: enzyme, trypsin; taxonomy, Insecta (749,851 sequences); fixed modification, carbamidomethylation (C); variable modifications, deamidated (NQ), methionine oxidation (M); protein mass, unrestricted; peptide mass tolerance, $\pm 60-100 \mathrm{ppm}$; fragment mass tolerance, $\pm 0.3 \mathrm{Da}$; and one missed cleavage allowed. For presentation of data, the taxonomy Insecta was selected due to the fact that some proteins had highest scores for some other insects. Hits scored with $P<0.05$ were considered significant.

\subsection{Semiquantitative evaluation of $2 \mathrm{D}$-Es of emerged bees with red-eye pupa}

To compare the 2D-E hemolymph of emerged honeybees with the pupal stage, we adopted the results from our previous study (Erban et al. 2014) of red-eye pupal hemolymph performed on the same apparatus using the same methods and identical $20-\mu \mathrm{L}$ hemolymph volumes. Because the patterns of the 2D-Es of the emerged bees and red-eye pupae were found to be very different, we evaluated the data at a semiquantitative level only. The data are presented as the presence or absence of a protein in the respective developmental stage, or lower, higher, or of similar abundance. Dymension software (ver. 3.0.1.4; Syngene, Cambridge, UK) was used to determine the number of spots and a normalized volume of the Idgf4 protein spot in the 2D-Es in the two developmental stages. The settings of the spot analyzer were as follows: (i) Spot Analysis: Gaussian limit threshold-0.05; minimum Gaussian quality index-0.1, minimum significance - 100, maximum Gaussians per patch-3, minimum fit probability-0.01; (ii) Patch Analyzer: blur radius - 1.2 , detection confidence ratio-30, separation confidence ratio-10, peak limit threshold -0.01 , splitting threshold -0.02 ; and (iii) Gaussian detector: maximum distance squared to center- 1.5 , minimum radius.

\section{RESULTS}

\subsection{Results of 2D-E mapping of newly emerged worker bee hemolymph}

Representative images of the analyzed 20 or $30 \mu \mathrm{L}$ of hemolymph volumes of $2 \mathrm{D}-\mathrm{E}$ Coomassie-stained, $14 \%$ SDS-PAGE, pI 310, and 4-7 IEF gels are presented in Figures 1 and $2 \mathrm{a}-\mathrm{c}$, respectively; see also the Supplemental Material Tables S1 and S2 for the list of identified proteins, with details on identification and characterization, respectively. The use of a pI 4-7 IEF separation in 2D-E analyses greatly improved the separation of accumulated protein spots, enabling 
3

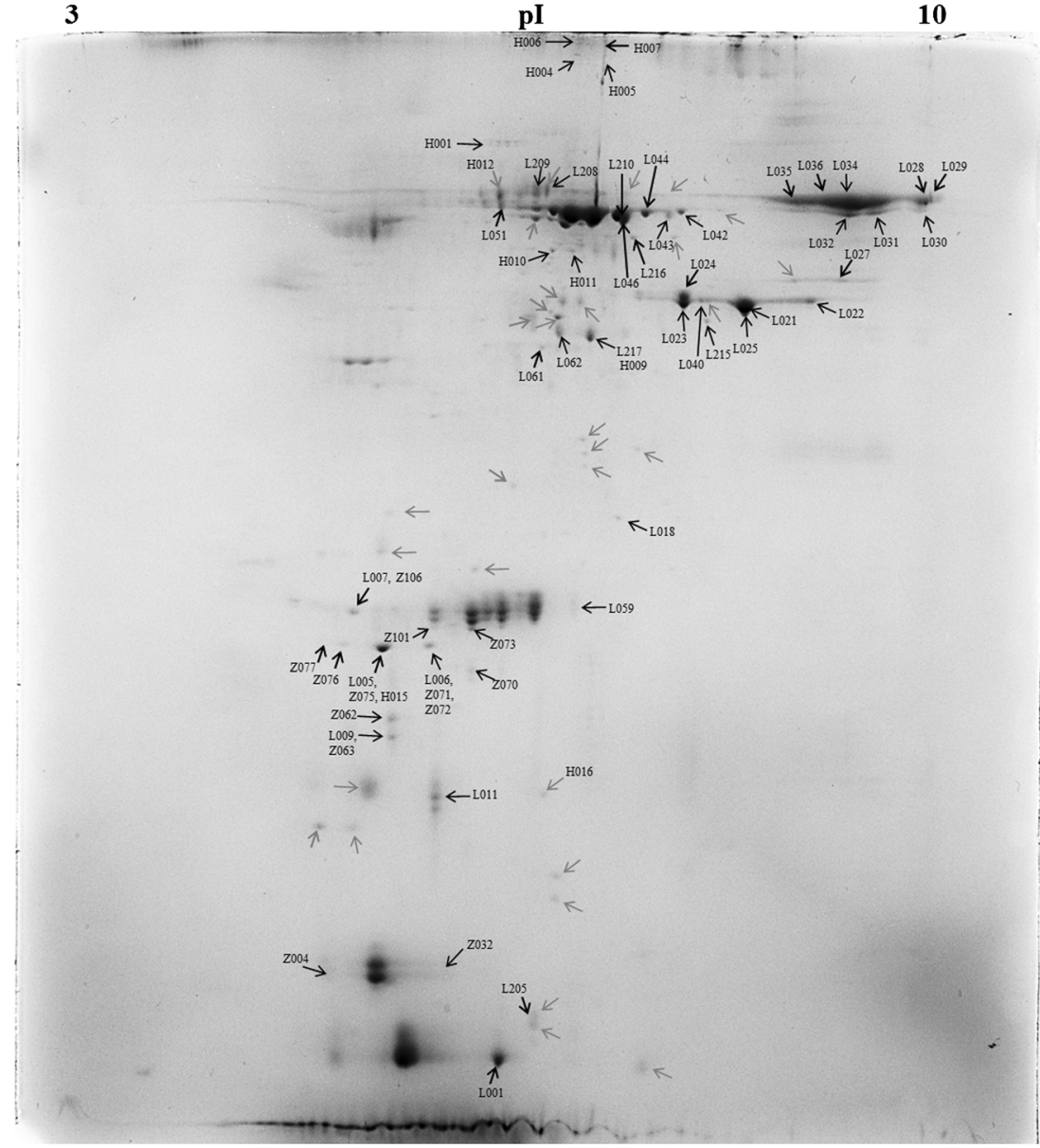

Figure 1. Representative 2D-E image of a pI 3-10, $14 \%$ SDS-PAGE analysis with marked identified protein spots of honeybee worker hemolymph collected at the time of emergence. The 2D-E image results from a $30-\mu \mathrm{L}$ hemolymph analysis of a single individual. See Table S1 and S2 for the list of identified proteins with details of MS/MS identifications. See also Figure S1 for the 2D-E figures pI 3-10 on $14 \%$ SDS-PAGE generated from a 20$\mu \mathrm{L}$ hemolymph; Figure S1 shows three biological replicates.

us to easily distinguish isoforms of the proteins.

Significant $(P<0.05)$ MALDI TOF/TOF results were protein scores greater than 71 . Identifications of some proteins had highest scores to species different from A. mellifera, e.g., glutathione S-transferase sigma class or ferritin. Protein identifications to ferritin heavy chain [A. cerana cerana] (JF330112.1; gi|357372779) and ferritin light chain [A. cerana cerana] (JF340051.1; gi|357372804) apparently correspond to A. mellifera ferritin light and heavy chains, respectively. This was confirmed by
Blast searches and the position of ferritin spots in 2D-E (Figure 2c).

\subsection{Results of the comparison of 2D-Es of newly emerged worker bees and red- eye pupae}

The analysis via the Dymension software demonstrated a low amount in the number of spots and decreased protein abundance in the emerged bee hemolymph compared to the red-eye pupal hemolymph (Table I). The number of visible hemolymph protein spots 

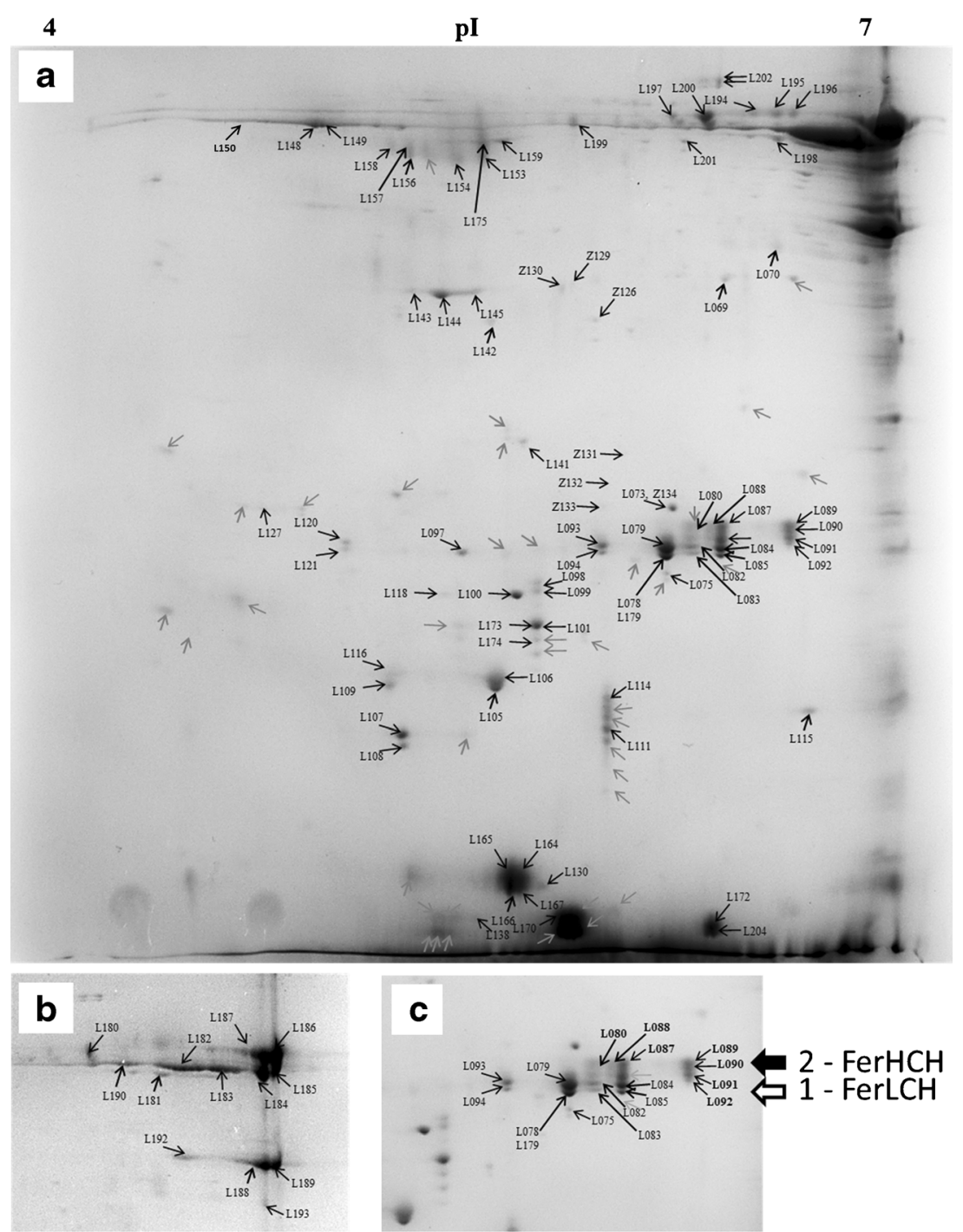

Figure 2. Representative 2D-E image of a pI 4-7, $14 \%$ SDS-PAGE analysis with marked identified protein spots of honeybee worker hemolymph collected at the time of emergence. a The 2D-E image was generated from $30 \mu \mathrm{L}$ of hemolymph of a single individual. b Details on high molecular weight proteins from $20 \mu \mathrm{L}$ of hemolymph. c Details showing isoforms of ferritin heavy chain $(\mathrm{FerHCH})$ and ferritin light chain (FerLCH). See Table S1 and S2 for the list of identified proteins with details about the MS/MS identifications.

was 4.54 times lower in the newly emerged bee compared to the red-eye pupa, as indicated by the consensus spots detected in the 2D-E (Table I). Chitinase-like protein Idgf4 and its isoforms had the same quantity in both stages. Therefore, the spot (spot no. L021 in Figure 1) corresponding to Idgf4 in the samples of the two stages (see Figure S1 and S2 for detail) was used to infer on quantitative changes via a normalized volume (Table I). The normalized volume of the Idgf4 spot was 4.48 times higher in emerged bee hemolymph compared to red-eye pupa.

Comparing the 2D-E pI 3-10, $14 \%$ SDSPAGE of $20-\mu \mathrm{L}$ hemolymph of newly emerged bees with $20-\mu \mathrm{L}$ red-eye pupal hemolymph 
Table I. Consensus spots and normalization volumes of selected chitinase-like protein Idgf4 spots determined by Dymension software.

\begin{tabular}{lll}
\hline Sample no./stage & Consensus spots & Norm. vol. of Idgf4 spot \\
\hline 1/emerged bee & 387 & 1.677 \\
2/emerged bee & 317 & 1.696 \\
3/emerged bee & 367 & 1.643 \\
Average \pm SD of 1-3 & $357 \pm 36$ & $1.672 \pm 0.027$ \\
4/red-eye pupa & 1383 & 0.436 \\
5/red-eye pupa & 1669 & 0.296 \\
6/red-eye pupa & 1807 & 0.388 \\
Average \pm SD of 4-6 & $1620 \pm 216$ & $0.373 \pm 0.071$ \\
\hline
\end{tabular}

Three independent biological replicates from both red-eye pupae and newly emerged bees were aligned; see Figures S1 and S2 for the figures used in the analysis and a demonstration of the analysis using Dymension. Chitinase-like protein Idgf4 was found to be constant in both stages. The results showed a 4.54-fold decrease in the number of spots after transition from the red-eye pupal stage to the newly emerged bee. The 4.48-fold lower normalized volume of the Idgf4 spot in red-eye pupal hemolymph compared to emerged bee hemolymph indicated a corresponding decrease in protein in the hemolymph

(Figure 3, Table II), clearly different 2D-E patterns were obtained. The protein spots were analyzed using MALDI TOF/TOF in the two stages

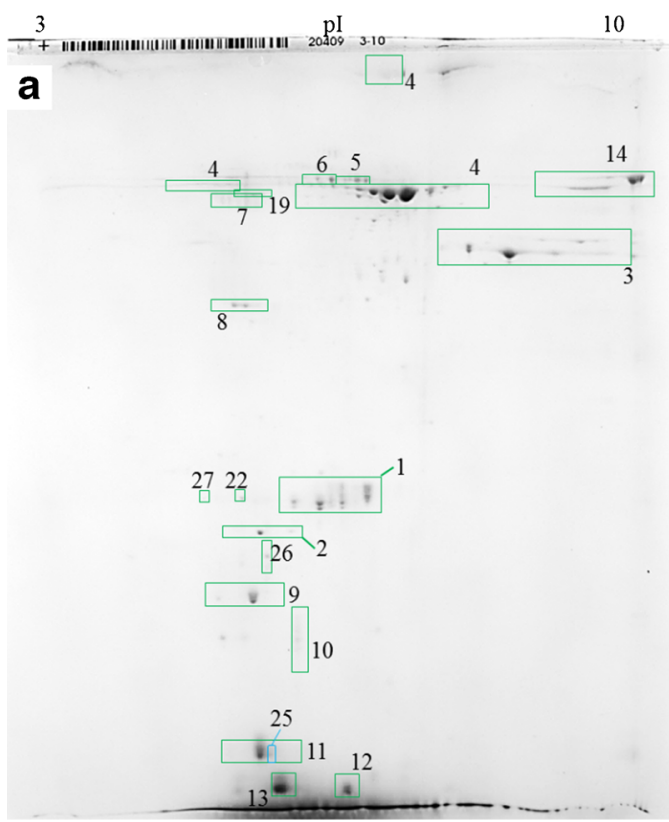

independently, and at the same respective position, the results matched. Some major proteins were not detected in the 2D-E of newly emerged

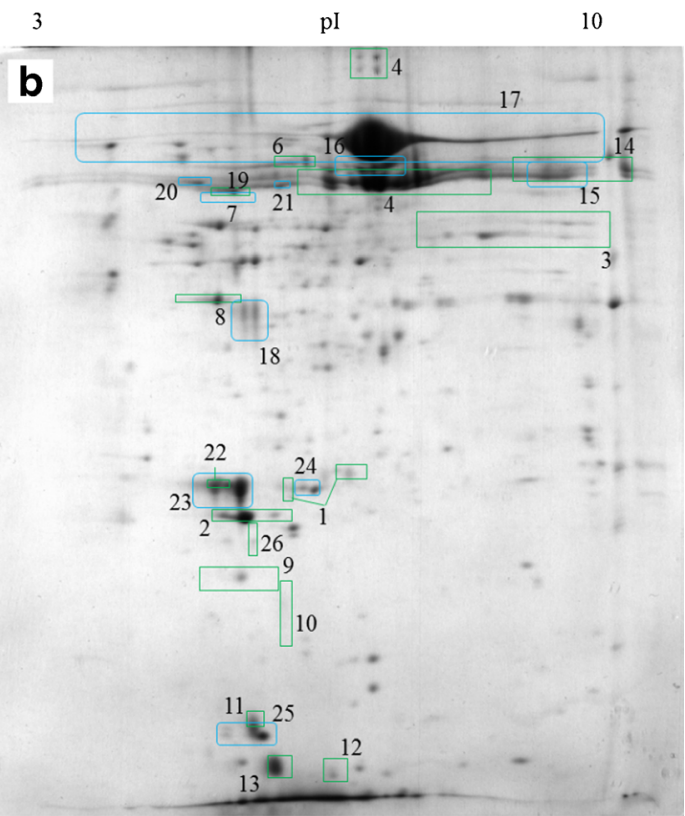

Figure 3. Comparison of 2D-E proteome maps (pI 3-10; $14 \%$ SDS-PAGE) generated from $20 \mu \mathrm{L}$ hemolymph of newly emerged worker bees (a) and red-eye pupae (b ). The 2D-E image of the red-eye pupal stage was reproduced from our previous study (Erban et al. 2014). Selected proteins are marked in the figure. See Table II for the list of protein groups marked. See Figure S1 for the 2D-E figures of the three biological replicates generated from $20 \mu \mathrm{L}$ of newly emerged bee hemolymph and Figure $\mathrm{S} 2$ for the 2D-E figures of the three biological replicates generated from $20 \mu \mathrm{L}$ of red-eye pupal hemolymph. 
Table II. Semiquantitative evaluation of major and selected changes between red-eye pupal and newly emerged bee hemolymph.

\begin{tabular}{|c|c|c|c|c|}
\hline No. & Protein name & $\begin{array}{l}\text { Newly } \\
\text { emerged bee }\end{array}$ & $\begin{array}{l}\text { Red-eye } \\
\text { pupa }\end{array}$ & $\begin{array}{l}\text { Semiquantitative } \\
\text { neb/rep }\end{array}$ \\
\hline 1 & Ferritin & Yes & Yes & Higher \\
\hline 2 & GST sigma class & Yes & Yes & Lower \\
\hline 3 & Chitinase-like protein Idgf4-like & Yes & Yes & Similar \\
\hline 4 & Hexamerin $70 \mathrm{a}$ & Yes & Yes & Similar \\
\hline 5 & Toll-like & Yes & No & - \\
\hline 6 & Alpha glucosidase & Yes & Yes & Similar \\
\hline 7 & $\begin{array}{l}\text { LOC413792; fibrillin; peroxisomal } N(1) \text {-acetyl-spermine/ } \\
\text { spermidine oxidase-like isoform }\end{array}$ & Yes & No & - \\
\hline 8 & Actin & Yes & Yes & Lower \\
\hline 9 & Apolipophorin III & Yes & Yes & Similar \\
\hline 10 & NPC2 homolog & Yes & Yes & Similar \\
\hline 11 & OBP14 & Yes & Yes & Similar \\
\hline 12 & Chemosensory protein 3 precursor & Yes & Yes & Higher \\
\hline 13 & OBP13 & Yes & Yes & Similar \\
\hline 14 & Apolipophorins & Yes & Yes & Similar \\
\hline 15 & Hexamerin $70 \mathrm{c}$ & No & Yes & - \\
\hline 16 & Hexamerin $70 b$ & No & Yes & - \\
\hline 17 & Hexamerin 110 & No & Yes & - \\
\hline 18 & Beta-ureidopropionase & No & Yes & - \\
\hline 19 & Heat shock protein cognate 4 & Yes & Yes & Lower \\
\hline 20 & Heat shock protein cognate 3 & No & Yes & - \\
\hline 21 & Heat shock protein cognate 5 & No & Yes & - \\
\hline 22 & Heat shock protein beta-1 & Yes & Yes & Lower \\
\hline 23 & Short-chain dehydrogenase/reductase & No & Yes & - \\
\hline 24 & Peroxiredoxin-like protein & No & Yes & - \\
\hline 25 & FABP-like protein & Yes & Yes & Lower \\
\hline 26 & Phospholipid hydroperoxide glutathione peroxidase & Yes & Yes & Similar \\
\hline 27 & Circadian clock-controlled protein & Yes & No & - \\
\hline
\end{tabular}

See Figure 3 for the comparison of marked protein groups in the 2D-Es. Legend: Yes - the protein was detected in 2D-Es of the stage; No - the protein was detected in 2D-Es of the stage; dash (-) - semiquantitative evaluation was not applicable as the protein was not detected in one type of the examined samples = qualitative change

bees compared to red-eye pupae, or vice versa (Table II).

\section{DISCUSSION}

Comparison of both the consensus spots and normalized volume of the selected Idgf4 spot demonstrated considerable protein depletion at the time of a bee's emergence compared to the red-eye pupal stage. At emergence, the hemolymph is low in protein, probably due to extensive protein depletion (metabolic use) during the non-feeding period associated with body reconstruction. Therefore, the proteins at the time of emergence represent a minimal need before the first feeding allows the synthesis of new proteins. Some major proteins were not detected in the 2DE of newly emerged workers compared to red-eye pupae, e.g., a short-chain dehydrogenase reductase; storage hexamerins $110,70 \mathrm{~b}$, and $70 \mathrm{c}$; beta- 
ureidopropionase; and some heat shock proteins or peroxiredoxin-like proteins. On the other hand, some proteins were detected solely in the 2D-E of emerged bees, e.g., a circadian clock-controlled protein and toll-like receptor 13 . Thus, the changes in hemolymph proteins are largely at a qualitative level. The presence of proteins at similar abundance (hexamerin 70a, Idgf4) indicated its equal need in the red-eye pupae and in the newly emergent worker bee. The protein composition of the hemolymph of newly emerged bees, i.e., relatively high levels of ferritin, glutathione S-transferase, or toll-like receptor 13 , indicates preconditioning to new stimuli, i.e., stress from a new environment or pathogen, after leaving the capped comb, which is a closed environment. The major groups of proteins detected in our analysis are discussed below; the remainder of the detected proteins is listed in Table $\mathrm{S} 1$.

\subsection{Chitinase-like protein Idgf4 is constant}

Idgf4 is considered a developmental protein in A. mellifera larva (Li et al. 2010; Feng et al. 2014). Feng et al. (2014) found Idgf4 to be up-regulated in the hemolymph of both A. mellifera and A. cerana from the larval to the pupal stage (Feng et al. 2014). Relatively strong spots of Idgf4 were identified in winter (Erban et al. 2013) and summer (Bogaerts et al. 2009) honeybee hemolymph. Even though Idgf4 is apparently present (used) throughout the life cycle of honeybee workers, its exact functions remain unknown. In Drosophila, Idgfs stimulate the proliferation, polarization, and motility of imaginal disc cells in cooperation with insulin (Kawamura et al. 1999). According to Butler et al. (2003), Idgf4 is expressed in the peripodial membrane (primarily in dorsal cells) and, presumably, the secretion of Idgf4 influences the development of the columnar epithelium (Butler et al. 2003). The constant concentration of Idgf4 indicated its equal need in the red-eye pupa and in the emerged worker hemolymph. Moreover, Idgf4 could be used as a quantitative reference for other protein spots.

\subsection{Hexamerin-storage or transport protein?}

Hexamerins are principally storage proteins that serve as a source of energy and amino acids in non-feeding periods. Overall, four hexamerin genes, Hex70a, Hex70b, Hex70c, and Hex110, were identified in the honeybee genome (Martins et al. 2010). Protein products of these genes were identified in the hemolymph of different stages of the honeybee via 1D-E (Danty et al. 1998; Cunha et al. 2005; Martins and Bitondi 2012). All of the four hexamerins have been identified by 2D-E in red-eye pupal hemolymph (Erban et al. 2014). In the 2D-E analyses of newly emerged honeybee workers, we successfully identified protein products of only hexamerin 70a. The disappearance of hexamerins $110,70 \mathrm{~b}$, and $70 \mathrm{c}$ indicates that they exerted a function during metamorphosis, while at the time of emergence, their function ended.

According to Martins et al. (2010), all of the four genes are highly transcribed by the larval fat body, while only $70 \mathrm{a}$ and 110 have detectable expression in the adult fat body. According to Cunha et al. (2005), a four-band hexamerin pattern found in the larval stage is maintained without apparent modification until the middle of the pupal stage, when melanin pigments appear in the thoracic cuticle, thus characterizing the light pigmented pupal phase; the hexamerin 110 band becomes barely visible, whereas the hexamerin $70 \mathrm{a}, 70 \mathrm{~b}$, and 70c subunits were still perfectly detectable. Subsequently, i.e., at the transition to adult life, hexamerins $70 \mathrm{~b}$ and $70 \mathrm{c}$ progressively decrease in the hemolymph, but they were still visible at the 7 th day of adult life. The abundance of the hexamerin 70a subunit does not apparently change, at least until the 7th day of adult life (Cunha et al. 2005). Danty et al. (1998) showed that hexamerin $70 \mathrm{a}$ is the only hexamerin present in a large amount in hemolymph of later adult honeybee worker stages. Hexamerins $70 \mathrm{~b}$ and 70c exhibit a similar developmental profile because they disappear from the hemolymph with worker emergence, while hexamerin 110 disappears earlier than 70b and 70c (Danty et al. 1998). Bitondi et al. (2006) found that hexamerin 110 subunits are highly abundant in larval hemolymph, but are decreased at the larval spinning 
stage, and remain at low levels in pupae and adults (Bitondi et al. 2006).

We conclude that our findings using 2D-E proteomic analysis clarify previous results that the hexamerins $110,70 \mathrm{~b}$, and $70 \mathrm{c}$ are depleted from the honeybee worker hemolymph at the time of emergence, while only hexamerin 70 a remains present (functional) at a higher concentration. The continued presence of hexamerin $70 \mathrm{a}$ in honeybee hemolymph at the time of emergence indicates that it is not used primarily as a storage protein but exerts another function and may serve a transport function.

\subsection{Ferritin-a high abundance protein in newly emerged honeybees}

rOur results indicate that isoforms assigned as NCBInr record $A$. mellifera PREDICTED: ferritin heavy chain (XP_624076.1; gi|66524161) are of higher molecular weight than those that were identified as NCBInr record A. mellifera PREDICTED: ferritin subunit (XP_624044.3; gi|328782011). Thus, the honeybee ferritins are present in the $\mathrm{H}$ and the L type. We showed that the ferritin level at the time of emergence increased in hemolymph compared with pupal hemolymph. In vertebrates, elevated serum ferritin levels have been reported in a number of pathological states (Morikawa et al. 1995), and ferritin acts as an immunosuppressor, signaling molecule and immune regulator (Recalcati et al. 2008). In Drosophila, overexpression of both the $\mathrm{HCH}$ and $\mathrm{LCH}$ genes is required to confer protection against oxidative stress (Missirlis et al. 2007). At the end of metamorphosis and at the time when the honeybee adult leaves the stable environment of the comb cell, the individual is put under increased stress, and the immune system will start to adapt to the new environment. Therefore, it is possible that the high level of ferritin in the emerged honeybee hemolymph is an important component of the immune system and may regulate the honeybee immune system so as not to overreact to the new stimulus outside the brood cell.

\subsection{Glutathione S-transferase sigma class}

In general, glutathione S-transferases (GSTs) are enzymes connected to the antioxidative defense system that eliminates reactive oxygen species generated as by-products of aerobic metabolism (Corona and Robinson 2006). Sigma GSTs have evolved specialized functions and have both anti- and pro-inflammatory functions, depending on the type of immune response (Flanagan and Smythe 2011). Sigma GSTs have been detected in different honeybee developmental stages, showing that this enzyme is ubiquitous throughout the entire life cycle of the honeybee (Erban et al. 2014). The pivotal role as a protectant against the by-products of oxidative stress during honeybee metamorphosis has been suggested (Erban et al. 2014). The abundance of Sigma GST partially decreased compared with pupal hemolymph. However, the still considerable presence suggests an apparent importance of the enzyme for the emerging honeybee. Apparently, these enzymes play an important, whether protective or not, role in the emerging honeybee, as well as in the pupa.

\subsection{Olfaction-Odorant-binding and chemosensory proteins}

Chemosensory proteins (CSPs) are similar to odorant-binding proteins (OBPs) and share some of their characteristics, but CSPs belong to an evolutionarily distinct lineage (Foret et al. 2007). We detected Obp13, Obp14, and CSP3 in the hemolymph of newly emerged worker bees, similar to that from red-eye pupae (Erban et al. 2014). While the abundance of OBPs was similar in both stages, CSP3 increased at the time of emergence. Furthermore, Feng et al. (2014) detected Obp13, Obp14, and CSP3 in hemolymph of A. mellifera and $A$. cerana bees. Zheng et al. (2011) detected Obp13 and 14 during A. mellifera pupal head development. Obp13, 14, 15, and chemosensory protein 3 (ASP3) were identified in the hemolymph of $A$. mellifera summer workers (Bogaerts et al. 2009), and Obp14 was found in winter worker hemolymph (Erban et al. 2013). Briand et al. (2002) observed that CSP3 is a brood pheromone carrier in A. mellifera. Foret et al. 
(2007) found CSP3 in late larvae, but no detectable expression of CSP3 was found during the initial stages of pupal development (24 h); however, the CSP3 transcripts were, again, detectable in red-eye pupae, and the level gradually increased as the head became dark. This result suggested that CSP3 plays a role in cuticle maturation, and its binding properties are not limited only to brood pheromone recognition, but may also transport hydrophobic cuticular hydrocarbons. Thus, it was inferred that CSPs are multifunctional, context-dependent proteins involved in diverse cellular processes ranging from embryonic development to chemosensory signal transduction; some CSPs may function in cuticle synthesis, consistent with their evolutionary origins in arthropods (Foret et al. 2007).

Our results are consistent with other works that found Obp13, Obp14, and CSP3 as abundant hemolymph proteins during worker bee development. We demonstrated that CSP3 was increased in newly emerged bees compared to red-eye pupae, supporting the multifunctional suggestions by Foret et al. (2007). Still, according to the binding capacity of these proteins for hydrophobic compounds, it seems possible that they function as transport proteins in the hemolymph (Erban et al. 2014).

\subsection{Lipid transport and metabolism}

Proteins involved in lipid transport and metabolism detected in emerged honeybee hemolymph were apolipophorins, apolipophorin III, and fatty acid-binding protein (FABP). Lipophorins are the principal lipoproteins in insect hemolymph that transport fats and other hydrophobic compounds (Robbs et al. 1985). Lipophorins are composed of $\sim 250$-kDa apolipophorin-I (apoLp-I), $\sim 80-\mathrm{kDa}$ apolipophorin-II (apoLp-II), and small MW $\sim 17$ $\mathrm{kDa}$ apolipophorin-III (apoLp-III) associated with the higher MW apoLPs (Robbs et al. 1985). In newly emerged bees, we detected apoLPs of similar abundance as in the red-eye pupal stage. The function of prototypical, exchangeable apolipoprotein apoLp-III in the adult life stage is to transport diacylglycerol from the fat body lipid storage depot to flight muscles (Wang et al. 2002). This multifunctional insect protein can stimulate antimicrobial peptide production in insect hemolymph and enhance phagocytosis in insect blood cells (hemocytes), and may act as a pattern recognition molecule for multiple microbial and parasitic invaders (Whitten et al. 2004). FABPs are 14-15-kDa proteins that bind reversibly to hydrophobic ligands with high affinity, such as saturated and unsaturated long-chain fatty acids and other lipophilic substances including eicosanoids and retinoids (Furuhashi and Hotamisligil 2008). FABPs are also known to be linked to inflammatory pathways (Makowski and Hotamisligil 2004) and are thought to be connected to the insulin/ insulin-like signaling network, which is conserved among animals and is central to growth and development (Wolschin et al. 2011). FABPs were found to be continuously highly expressed in honeybee worker larvae, but showed decreased expression in adults (Randolt et al. 2008), suggesting age-dependent decreases in the worker hemolymph. Here, we found FABPs to be of low abundance in the emerged bee hemolymph compared to pupa. It confirms decreased need of FABPs for adult bee after metamorphosis.

\subsection{Heat shock proteins}

Heat shock proteins were also detected in the emerged bee hemolymph; however, they were of low abundance compared to the pupal stage. The two heat shock proteins that were detected were heat shock protein beta- 1 and heat shock cognate 4. The HSP expression levels were originally found to increase under exposure to heat shock or other stressors. These proteins are constitutively synthesized and mediate many cellular processes by influencing and supporting a higher order of protein structure (Liang and MacRae 1997). Therefore, the higher abundance of HSPs in pupal hemolymph compared to the newly emerged honeybee stage is appropriate for both the greater needs of protein formation and the increased stress during metamorphosis.

\subsection{Toll-like 13}

Toll-like receptor 13 contains leucine-rich repeats (LRRs). LRRs are 20-29 residue sequence motifs present in many proteins that participate in 
protein-protein interactions and have different functions and cellular locations (Marchler-Bauer et al. 2015). TLRs are important initiators of innate immunity and recognize diverse microbial products that are collectively known as pathogen-associated molecular patterns. Signaling pathways are then activated, which culminate in the induction of pro-inflammatory proteins that trigger both innate and adaptive immunity (Doyle and O'Neill 2006). They are known to play an important role in insect immunity and development (Evans et al. 2006). Some TLRs were found to be involved in insect intracellular signal transduction and the initiation of the insect antimicrobial immune response (Aronstein and Saldivar 2005; Evans et al. 2006; Flenniken and Andino 2013). Future observations of toll-like 13 after an immune challenge could demonstrate its function in the pathogen reorganization pathway. The presence of this protein in the hemolymph of newly emerged bees could be connected to the immune cells because they were not separated during sample processing in this study.

\subsection{Circadian clock-a signal of adult eclosion}

Circadian clock-controlled protein belongs to a family of hemolymph juvenile hormone-binding proteins (Marchler-Bauer et al. 2015). This protein has been found to be abundantly expressed during pupation and to decrease rapidly with age. In Drosophila, its expression was found to be under pupal (eclosion) circadian clock control (Lorenz et al. 1989). Our detection of this protein in the emerged bee hemolymph and its lack in redeye pupae could mean that this protein is connected to the molt from the pupal stage and transition to the adult bee.

\subsection{Other proteins}

Furthermore, the proteins antithrombin-III, dipeptidase 1-like, phospholipid hydroperoxide glutathione peroxidase, mitochondrial isoform 1, neutral alpha-glucosidase AB-like isoform 2, chitotriosidase-1-like, arginine kinase, alpha-glucosidase, fibrillin-1, fibrillin-2-like, 14-3-3 zeta, and actin were detected in the hemolymph of newly emerged bees.

\subsection{Methodical note: sample processing for proteomic analysis}

In this study, we did not process the hemolymph before the analysis and also omitted the use of protease inhibitors. In addition, we compared 2D-Es generated from identical volumes of honeybee hemolymph. As demonstrated in this and our previous study (Erban et al 2014), the cleanup step is not necessary to obtain good 2DE separation of honeybee hemolymph proteins, even if a high protein load is applied. Although the sample cleanup can improve separation of some proteins, it can lead to the loss of some proteins and is time-consuming. Therefore, the benefit of omitting the sample cleanup step would be increased reproducibility and simplification of the method. Next, protease inhibitors were not needed because the samples were dissolved in DeStreak, which contains urea and thiourea, causing denaturation of proteins. A critical point in the entire analysis is quality and speed of hemolymph collection before the sample is frozen and/or mixed to DeStreak (or similar solution). The hemolymph can be frozen on dry ice after collection or directly mixed with DeStreak and then frozen.

\section{CONCLUSIONS}

We have generated a 2D-E protein profile of A. mellifera worker hemolymph at the time of emergence from the brood cell. In addition, the 2D-E profile of emerged worker bees was compared with the previously analyzed $2 \mathrm{D}-\mathrm{E}$ results of red-eye pupal hemolymph. Our results demonstrated a large protein depletion during the nonfeeding period and showed proteins that were present (functional) at the time of the bee's emergence. The complete depletion of hexamerins 110, $70 \mathrm{~b}$, and $70 \mathrm{c}$ puts in evidence their storage function, while the continuous presence of hexamerin $70 \mathrm{a}$ is consistent with a transport function in hemolymph. The disappearance of proteins, such as short-chain dehydrogenase reductase or betaureidopropionase, indicates their crucial functions during the pupal stage. The high abundance of 
ferritin in the hemolymph and the detection of circadian clock or toll-like proteins were interesting. We expect that future studies investigating protein changes during honeybee development and disease-related studies at the individual or population level will benefit from our results. The generated 2D-E map can also be used to investigate $V$. destructor physiology and hostparasite interactions, because the pupal hemolymph serves as the sole food source for this major honeybee pest.

\section{ACKNOWLEDGMENTS}

This study was supported by a grant from the Grant Agency of the Ministry of Agriculture of the Czech Republic (http://eagri.cz/public/web/mze/), Grant Nos. QJ1310085 and RO0415. We would like to thank Lenka Matuskova for technical help. The authors would like to thank the anonymous reviewers and editor for their valuable comments and suggestions that have improved the manuscript.

Cartographie détaillée du protéome de l'hémolymphe d'ouvrières d'abeilles venant d'émerger et comparaison avec celui du stade nymphal "yeux rouges"

\section{Apis mellifera/ hémolymphe/ métamorphose/ hexamérine/ ferritine}

Ein detailierter Vergleich der Proteome der Hämolymphe von frischgeschlüpften Arbeiterinnen der Honigbiene mit der des rotäugigen Puppenstadiums

\section{Apis mellifera / Hämolymphe / Metamorphose / Hexamerin / Ferritin}

\section{REFERENCES}

Aronstein, K., Saldivar, E. (2005) Characterization of a honey bee Toll related receptor gene Am18w and its potential involvement in antimicrobial immune defense. Apidologie 36 (1), 3-14

Bitondi, M.M.G., Nascimento, A.M., Cunha, A.D., Guidugli, K.R., Nunes, F.M.F., Simoes, Z.L.P. (2006) Characterization and expression of the Hex 110 gene encoding a glutamine-rich hexamerin in the honey bee, Apis mellifera. Arch. Insect Biochem. Physiol. 63 (2), $57-72$
Bogaerts, A., Baggerman, G., Vierstraete, E., Schoofs, L., Verleyen, P. (2009) The hemolymph proteome of the honeybee: gel-based or gel-free? Proteomics 9 (11), 3201-3208

Briand, L., Swasdipan, N., Nespoulous, C., Bezirard, V., Blon, F., Huet, J.-C., Ebert, P., Pernollet, J.C. (2002) Characterization of a chemosensory protein $(\mathrm{ASP} 3 \mathrm{c})$ from honeybee (Apis mellifera L.) as a brood pheromone carrier. Eur. J. Biochem. 269 (18), 4586-4596

Butler, M.J., Jacobsen, T.L., Cain, D.M., Jarman, M.G., Hubank, M., Whittle, J.R.S., Phillips, R., Simcox, A. (2003) Discovery of genes with highly restricted expression patterns in the Drosophila wing disc using DNA oligonucleotide microarrays. Development 130 (4), 659-670

Chan, Q.W.T., Foster, L.J. (2008) Changes in protein expression during honey bee larval development. Genome Biol. 9 (10), R156, DOI: 10.1186/gb-2008-9$10-\mathrm{r} 156$

Chan, Q.W.T., Howes, C.G., Foster, L.J. (2006) Quantitative comparison of caste differences in honeybee hemolymph. Mol. Cell. Proteomics 5 (12), 2252-2262

Corona, M., Robinson, G.E. (2006) Genes of the antioxidant system of the honey bee: annotation and phylogeny. Insect Mol. Biol. 15 (5), 687-701

Cunha, A.D., Nascimento, A.M., Guidugli, K.R., Simoes, Z.L.P., Bitondi, M.M.G. (2005) Molecular cloning and expression of a hexamerin cDNA from the honey bee, Apis mellifera. J. Insect Physiol. 51 (10), 1135-1147

Danty, E., Arnold, G., Burmester, T., Huet, J.-C., Huet, D., Pernollet, J.-C., Masson, C. (1998) Identification and developmental profiles of hexamerins in antenna and hemolymph of the honeybee, Apis mellifera. Insect Biochem. Mol. Biol. 28 (5-6), 387-397

Doyle, S.L., O'Neill L.A. (2006) Toll-like receptors: from the discovery of NFkappaB to new insights into transcriptional regulations in innate immunity. Biochem. Pharmacol. 72 (9), 1102-1113

Erban, T., Jedelsky, P.L., Titera, D. (2013) Twodimensional proteomic analysis of honeybee, Apis mellifera, winter worker hemolymph. Apidologie 44 (4), 404-418

Erban, T., Petrova, D., Harant, K., Jedelsky, P.L., Titera, D. (2014) Two-dimensional gel proteome analysis of honeybee, Apis mellifera, worker red-eye pupa hemolymph. Apidologie 45 (1), 53-72

Erban, T., Harant, K., Hubalek, M., Vitamvas, P., Kamler, M., Poltronieri, P., Tyl, J., Markovic, M., Titera, D. (2015) In-depth proteomic analysis of Varroa destructor: detection of DWV-complex, ABPV, VdMLV and honeybee proteins in the mite. Sci. Rep. 5, 13907, DOI: 10.1038/ srep 13907

Evans, J.D., Aronstein, K., Chen, Y.P., Hetru, C., Imler, J.L., Jiang, H., Kanost, M., Thompson, G.J., Zou, Z., Hultmark, D. (2006) Immune pathways and defence mechanisms in honey bees Apis mellifera. Insect Mol. Biol. 15 (5), 645-656 
Feng, M., Ramadan, H., Han, B., Fang, Y., Li, J.K. (2014) Hemolymph proteome changes during worker brood development match the biological divergences between western honey bees (Apis mellifera) and eastern honey bees (Apis cerana). BMC Genomics 15, 563, DOI: $10.1186 / 1471-2164-15-563$

Flanagan, J.U., Smythe, M.L. (2011) Sigma-class glutathione transferases. Drug Metab. Rev. 43 (2), 194-214

Flenniken, M.L., Andino, R. (2013) Non-specific dsRNAmediated antiviral response in the honey bee. PLoS One 8 (10), e77263, DOI: 10.1371/journal.pone.0077263

Foret, S., Wanner, K.W., Maleszka, R. (2007) Chemosensory proteins in the honey bee: insights from the annotated genome, comparative analyses and expressional profiling. Insect Biochem. Mol. Biol. 37 (1), 19-28

Furuhashi, M., Hotamisligil, G.S. (2008) Fatty acidbinding proteins: role in metabolic diseases and potential as drug targets. Nat. Rev. Drug Discov. 7 (6), 489 $-503$

Kawamura, K., Shibata, T., Saget, O., Peel, D., Bryant, P.J. (1999) A new family of growth factors produced by the fat body and active on Drosophila imaginal disc cells. Development 126 (2), 211-219

Li, J.K., Wu, J., Begna Rundassa, D., Song, F.F., Zheng, A.J., Fang, Y. (2010) Differential protein expression in honeybee (Apis mellifera L.) larvae: underlying caste differentiation. PLoS One 5 (10), e13455, DOI: 10.1371/journal.pone.0013455

Liang, P., MacRae, T.H. (1997) Molecular chaperones and the cytoskeleton. J. Cell Sci. 110 (13), 1431-1440

Lorenz, L.J., Hall, J.C., Rosbash, M. (1989) Expression of a Drosophila mRNA is under circadian clock control during pupation. Development 107 (4), 869-880

Makowski, L., Hotamisligil, G.S. (2004) Fatty acid binding proteins - The evolutionary crossroads of inflammatory and metabolic responses. J. Nutr. 134 (9), 2464S $-2468 \mathrm{~S}$

RMarchler-Bauer, A., Derbyshire, M.K., Gonzales, N.R., Lu, S., Chitsaz, F., Geer, L.Y., Geer, R.C., He, J., Gwadz, M., Hurwitz, D.I., Lanczycki, C.J., Lu, F., Marchler, G.H., Song, J.S., Thanki, N., Wang, Z., Yamashita, R.A., Zhang, D., Zheng, C., Bryant, S.H. (2015) CDD: NCBI's conserved domain database. Nucleic Acids Res. 43 (D1), D222-D226

Martins, J.R., Bitondi M.M.G. (2012) Nuclear immunolocalization of hexamerins in the fat body of metamorphosing honey bees. Insects 3 (4), 1039-1055

Martins, J.R., Nunes, F.M.F., Cristino, A.S., Simoes, Z.L.P., Bitondi, M.M.G. (2010) The four hexamerin genes in the honey bee: structure, molecular evolution and function deduced from expression patterns in queens, workers and drones. BMC Mol. Biol. 11, 23, DOI: 10.1186/1471-2199-11-23

Missirlis, F., Kosmidis, S., Brody, T., Mavrakis, M., Holmberg, S., Odenwald, W.F., Skoulakis, E.M.C., Rouault, T.A. (2007) Homeostatic mechanisms for iron storage revealed by genetic manipulations and live imaging of Drosophila ferritin. Genetics 177 (1), 89-100

Morikawa, K., Oseko, F., Morikawa, S. (1995) A role for ferritin in hematopoiesis and the immune system. Leuk. Lymphoma 18 (5-6), 429-433

Randolt, K., Gimple, O., Geissendorfer, J., Reinders, J., Prusko, C., Mueller, M.J., Albert, S., Tautz, J., Beier, H. (2008) Immune-related proteins induced in the hemolymph after aseptic and septic injury differ in honey bee worker larvae and adults. Arch. Insect Biochem. Physiol. 69 (4), 155-167

Recalcati, S., Invernizzi, P., Arosio, P., Cairo, G. (2008) New functions for an iron storage protein: the role of ferritin in immunity and autoimmunity. J. Autoimmun. 30 (1-2), 84-89

Robbs, S.L., Ryan, R.O., Schmidt, J.O., Keim, P.S., Law, J.H. (1985) Lipophorin of the larval honeybee, Apis mellifera L. J. Lipid Res. 26 (2), 241-247

Wang, J., Sykes, B.D., Ryan, R.O. (2002) Structural basis for the conformational adaptability of apolipophorin III, a helix-bundle exchangeable apolipoprotein. Proc. Natl. Acad. Sci. U. S. A. 99 (3), 1188-1193

Whitten, M.M.A., Tew, I.F., Lee, B.L., Ratcliffe, N.A. (2004) A novel role for an insect apolipoprotein (apolipophorin III) in beta-1,3-glucan pattern recognition and cellular encapsulation reactions. J. Immunol. 172 (4), 2177-2185

Winston, M.L. (1987) The Biology of the Honey Bee. Harvard University Press, Cambridge, MA, USA

Wolschin, F., Mutti, N.S., Amdam, G.V. (2011) Insulin receptor substrate influences female caste development in honeybees. Biol. Lett. 7 (1), 112-115

Woltedji, D., Fang, Y., Han, B., Feng, M., Li, R., Lu, X., Li, J. (2013) Proteome analysis of hemolymph changes during the larval to pupal development stages of honeybee workers (Apis mellifera ligustica). J. Proteome Res. 12 (11), 5189-5198

Zheng, A.J., Li, J.K., Begna, D., Fang, Y., Feng, M., Song, F.F. (2011) Proteomic analysis of honeybee (Apis mellifera L.) pupae head development. PLoS One 6 (5), e20428, DOI: 10.1371/ journal.pone.0020428 